\title{
PROOF OF THE FUNDAMENTAL GAP CONJECTURE
}

\author{
BEN ANDREWS AND JULIE CLUTTERBUCK
}

\section{INTRODUCTION}

We consider Schrödinger operators of the form $-\Delta+V$ with Dirichlet boundary conditions on a compact convex domain $\Omega$ in $\mathbb{R}^{n}$. The diameter of $\Omega$ is given by $D=\sup _{x, y \in \Omega}\|y-x\|$. We assume that the potential $V$ is semiconvex (i.e., $V+c\|x\|^{2}$ is convex for some $c$ ). Such an operator has an increasing sequence of eigenvalues $\lambda_{0}<\lambda_{1} \leq \lambda_{2} \leq \cdots$ and corresponding eigenfunctions $\left\{\phi_{i}\right\}_{i \geq 0}$ which vanish on $\partial \Omega$ and satisfy the equation

$$
\Delta \phi_{i}-V \phi_{i}+\lambda_{i} \phi_{i}=0
$$

The difference between the first two eigenvalues, $\lambda_{1}-\lambda_{0}$, is called the fundamental gap. It is of importance for several reasons: In quantum mechanics it represents the 'excitation energy' required to reach the first excited state from the ground state; it thus determines the stability of the ground state and so is also important in statistical mechanics and quantum field theory. The spectral gap also determines the rate at which positive solutions of the heat equation approach the first eigenspace, and it is through this characterization that we will prove the conjecture of the title.

It was observed by Michiel van den Berg $\mathrm{vdB}$ that for many convex domains, $\lambda_{1}-\lambda_{0} \geq 3 \pi^{2} / D^{2}$. This was also independently suggested by Ashbaugh and Benguria $\mathrm{AB}$ ] and Yau $\mathrm{Y2}$.

Gap Conjecture. Let $\Omega \subset \mathbb{R}^{n}$ be a bounded convex domain of diameter $D$, and $V$ a weakly convex potential. Then the eigenvalues of the Schrödinger operator satisfy

$$
\lambda_{1}-\lambda_{0} \geq \frac{3 \pi^{2}}{D^{2}} .
$$

In one dimension, the conjecture is settled. If $\Omega$ is an interval of length $D$, the eigenvalue equation (11) becomes a boundary value problem for an ODE: The eigenfunctions $\tilde{\phi}_{i}$ satisfy $\tilde{\phi}_{i}( \pm D / 2)=0$ and

$$
\tilde{\phi}_{i}^{\prime \prime}-\tilde{V} \tilde{\phi}_{i}+\mu_{i} \tilde{\phi}_{i}=0 \text { in }(-D / 2, D / 2) .
$$

For convenience we normalize these to have $\tilde{\phi}_{i}^{\prime}(D / 2)=-1$.

Received by the editors June 8, 2010 and, in revised form, July 9, 2010, and January 11, 2011. 2010 Mathematics Subject Classification. Primary 35P15, 35J10; Secondary 35K05, 58J35.

Key words and phrases. Parabolic equation, eigenvalue estimate, spectral gap.

This research was supported by Discovery Grant DP0985802 of the Australian Research Council.

(C)2011 American Mathematical Society Reverts to public domain 28 years from publication 
Ashbaugh and Benguria $\mathrm{AB}$ proved the conjecture for $n=1$ under slightly different assumptions: They considered symmetric single-well potentials, so that $\tilde{V}(x)=\tilde{V}(-x)$, and $\tilde{V}$ is nondecreasing on $[0, D / 2]$.

Theorem 1.1 (Ashbaugh-Benguria). If $n=1$ and $\tilde{V}$ is symmetric and single-well, then the eigenvalue gap satisfies

$$
\lambda_{1}-\lambda_{0} \geq \frac{3 \pi^{2}}{D^{2}}
$$

with equality attained when $\tilde{V}$ is constant.

Later, Horváth $[\mathrm{Ho}$ partly removed the symmetry assumption, allowing $\tilde{V}$ to be a single-well potential with minimum at the midpoint of the interval. In 1994, Lavine removed the assumption on the minimum point and proved the complete conjecture [La].

Theorem 1.2 (Lavine). The Gap Conjecture holds for $n=1$.

In higher dimensions, the conjecture has been proved for some highly symmetric cases: Bañuelos-Méndez-Hernández [BMH] (for $V \equiv 0$ ), Davis [D] and BañuelosKröger [BK] proved it for $\Omega \subset \mathbb{R}^{2}$ symmetric with respect to the $x$ and $y$ axes and convex in both $x$ and $y$.

Nonoptimal lower bounds have also been obtained: In an influential paper, Singer, Wong, Yau and Yau SWYY] used gradient estimates similar to those developed by $\mathrm{Li}[\mathrm{Li}$ ] and $\mathrm{Li}-\mathrm{Yau}[\mathrm{LY}$, together with the log-concavity of the first eigenfunction (proved for convex potentials by Brascamp and Lieb [BL, and also by different methods in [Ko] and in $[\mathrm{SWYY}]$ ) to show the gap is at least $\frac{\pi^{2}}{4 D^{2}}$. Yu and Zhong $\left[\mathrm{YZ}\right.$ improved this to $\frac{\pi^{2}}{D^{2}}$, following the work of Zhong and Yang [ZY] which similarly improved Li and Yau's estimate for the first nontrivial eigenvalue on a compact manifold [LY. Many other authors have used probabilistic arguments (including $[\mathrm{BL}$ and $[\mathrm{BK}]$ ). Such methods were used by Smits $[\mathrm{S}$ to recover the estimate of $\mathrm{Yu}$ and Zhong. It seems that any further improvement requires an improved estimate on the log-concavity of the first eigenfunction. Efforts in this direction have included work by Yau [Y3] and Ling $[\mathrm{L}$, who prove gap estimates depending explicitly on an upper bound for $D^{2}\left(\log \phi_{0}\right)$. The problem of nonconvex potentials has been studied by Yau [Y1. There is also considerable literature on potentials with double wells, including examples with arbitrarily small spectral gaps (see for example $\mathrm{Ha}$ ).

We should point out here that the fundamental gap for the Neumann problem (with zero potential) is simply the first nontrivial eigenvalue. On a convex domain, Payne and Weinberger [PW] proved that this is bounded below by $\pi^{2} / D^{2}$. Zhong and Yang $[\mathrm{ZY}]$ later reproduced the same result as a special case, using rather different methods.

We refer to the excellent survey by Ashbaugh [A] for further discussion of the fundamental gap conjecture and its history.

Our proof of the gap conjecture combines two ingredients, both of which involve analysis of the heat equation: The first ingredient is a method for estimating the modulus of continuity of solutions of parabolic equations which was developed in our earlier papers $\mathrm{AC} 1, \mathrm{AC} 2$. In this paper we say that a function $\eta$ on $\mathbb{R}_{+}$is a 
modulus of continuity for a function $f$ on $\Omega$ if

$$
|f(y)-f(x)| \leq 2 \eta\left(\frac{\|y-x\|}{2}\right)
$$

for all $x, y \in \Omega$, where $\|\cdot\|$ is the Euclidean. Here the factors of 2 are for later convenience. In [AC2, Theorem 4.1] we proved that if $u$ is any smooth solution of the heat equation with Neumann boundary data on a convex domain $\Omega$ of diameter $D$ in $\mathbb{R}^{n}$, then $u(., t)$ has modulus of continuity $\varphi(., t)$, where $\varphi$ is the solution to the one-dimensional heat equation on $[-D / 2, D / 2]$ with Neumann boundary data and initial data $\varphi(z, 0)=\frac{1}{2} \operatorname{osc} u(., 0) \operatorname{sgn}(z)$. The proof of this uses a maximum principle argument applied to a function of $2 n$ spatial variables and crucially exploits the full $2 n \times 2 n$ matrix of second derivatives. In that paper we were mostly concerned with the short-time application of this estimate to deduce Lipschitz bounds on $u$, but the estimate is also useful for large $t$ : One can show that $|\varphi(x, t)| \leq C \mathrm{e}^{-\frac{\pi^{2}}{D^{2}} t}$, so the oscillation of $u$ decays at an exponential rate, with exponent at least $\pi^{2} / D^{2}$. But if we choose $u$ to be the first nontrivial eigenfunction for the Neumann problem on $\Omega$, then $u(x, t)=u(x, 0) \mathrm{e}^{-\lambda t}$, where $\lambda$ is the corresponding eigenvalue. It follows that $\lambda \geq \frac{\pi^{2}}{D^{2}}$, and we recover the sharp estimate of Payne and Weinberger [PW]. In a separate work we will apply this method to more general eigenvalue estimates on manifolds.

As might be expected from the proof in SWYY, a second ingredient is required to apply this technique to the gap conjecture: The log-concavity of the first eigenfunction $\phi_{0}$. If we apply the method above using only the log-concavity of $\phi_{0}$, then we recover the estimate $\lambda_{1}-\lambda_{0} \geq \frac{\pi^{2}}{D^{2}}$, which is precisely the estimate of $\mathrm{Yu}$ and Zhong YZ. As noted above, it is clear that we must use something stronger than log-concavity to obtain a sharp result. Previous work $\mathrm{Y3}, \mathrm{L}$ produces stronger results assuming positive lower bounds on the Hessian of $\log \phi_{0}$, but such estimates cannot yield sharp results.

In trying to apply the modulus of continuity argument above, we are led to a log-concavity condition involving differences of derivatives of $\log \phi_{0}$.

We first introduce the relevant notions in a general context: If $\omega$ is a real function of a positive real variable, and $X$ is a vector field on a domain $\Omega$, we say that $\omega$ is a modulus of expansion for $X$ if for every $y \neq x$ in $\Omega$ we have

$$
\left(\left.X\right|_{y}-\left.X\right|_{x}\right) \cdot \frac{y-x}{\|y-x\|} \geq 2 \omega\left(\frac{\|y-x\|}{2}\right) .
$$

The factors of 2 are for later convenience. The name reflects the fact that a modulus of expansion implies a rate of increase of distances under the flow of $X$. We say that $\omega$ is a modulus of contraction for $X$ if the sign is reversed (that is, $-\omega$ is a modulus of expansion for $-X$ ). If $f$ is a semi-convex function on a domain $\Omega$, we say that $\omega$ is a modulus of convexity for $f$ if $\omega$ is a modulus of expansion for the gradient vector field $\nabla f$ of $f$, and $\omega$ is a modulus of concavity for $f$ if $\omega$ is a modulus of contraction for $\nabla f$. Note that $f$ is concave (convex) if and only if zero is a modulus of concavity (convexity) for $f$.

Our log-concavity estimates amount to an explicit modulus of concavity for $\log \phi_{0}$. Precisely, we assume that the potential $\tilde{V}$ is even, and that the potential $V$ in equation (1) is 'more convex' than $\tilde{V}$, in the sense that for any $y \neq x$ in 
$\Omega$ we have

$$
\left(\left.\nabla V\right|_{y}-\left.\nabla V\right|_{x}\right) \cdot \frac{(y-x)}{\|y-x\|} \geq 2 \tilde{V}^{\prime}\left(\frac{\|y-x\|}{2}\right) .
$$

That is, we assume that $\tilde{V}^{\prime}$ is a modulus of convexity for $V$. Under this assumption we prove in Theorem 1.5 that the first eigenfunction $\phi_{0}$ in (11) is 'more log-concave' than the first eigenfunction $\tilde{\phi}_{0}$ in (2), in the sense that

$$
\left(\left.\nabla \log \phi_{0}\right|_{y}-\left.\nabla \log \phi_{0}\right|_{x}\right) \cdot \frac{y-x}{\|y-x\|} \leq\left. 2\left(\log \tilde{\phi}_{0}\right)^{\prime}\right|_{z=\frac{\|y-x\|}{2}}
$$

for every $y \neq x$ in $\Omega$. That is, we prove that $\left(\log \tilde{\phi}_{0}\right)^{\prime}$ is a modulus of concavity for $\log \phi_{0}$. Note that if $V$ is convex, then the inequality (5) holds with $\tilde{V}=0$, and the modulus of concavity in (6) becomes $\left(\log \tilde{\phi}_{0}\right)^{\prime}(z)=-\frac{\pi}{D} \tan \left(\frac{\pi z}{D}\right)$. We will now list the main results.

Theorem 1.3 (Fundamental gap comparison theorem). If $V$ and $\tilde{V}$ are related by (51), then the spectral gap for $-\Delta+V$ on a convex domain $\Omega$ is bounded below by the spectral gap of the one-dimensional operator $-\frac{d^{2}}{d x^{2}}+\tilde{V}$ on $[-D / 2, D / 2]$.

If $V$ is convex, the gap conjecture follows since we can choose $\tilde{V}=0$ :

Corollary 1.4. If $V$ is weakly convex, then the spectral gap has the bound

$$
\lambda_{1}-\lambda_{0} \geq \frac{3 \pi^{2}}{D^{2}} .
$$

In particular, this applies for zero potential. The key step in proving the gap conjecture is a sharp log-concavity estimate for the first eigenfunction:

Theorem 1.5 (Log-concavity comparison theorem). Let $\phi_{0}$ be the first eigenfunction of (11). If the potential $V$ satisfies condition (5), then the log-concavity estimate (6) holds for every $y \neq x$ in $\Omega$.

Let us note a special case of this result which can be stated rather more elegantly: Suppose $\tilde{V}^{\prime}$ is also a modulus of convexity for $\tilde{V}$. It then follows from Theorem 1.5 applied with $n=1$ and $V=\tilde{V}$ that $\left(\log \tilde{\phi}_{0}\right)^{\prime}$ is a (sharp) modulus of concavity for $\log \tilde{\phi}_{0}$. This happens, for example, if $\tilde{V}$ has nonnegative third derivatives for positive arguments: In that case $\tilde{V}^{\prime}(b)-\tilde{V}^{\prime}(a) \geq \tilde{V}^{\prime}\left(\frac{b-a}{2}\right)-\tilde{V}^{\prime}\left(-\frac{b-a}{2}\right)=2 \tilde{V}^{\prime}\left(\frac{b-a}{2}\right)$, so that $\tilde{V}$ has (sharp) modulus of convexity $\tilde{V}^{\prime}$. It also happens if $\tilde{V}$ is the optimal choice defined by $2 \tilde{V}^{\prime}(z / 2)=\sup \{(\nabla V(x+z e)-\nabla V(x)) \cdot e: x, x+z e \in \Omega,\|e\|=1\}$. Thus in these cases we have:

Corollary 1.6. Under the conditions above, if the modulus of convexity of $V$ is bounded below by the modulus of convexity of $\tilde{V}$, then the modulus of concavity of $\log \phi_{0}$ is bounded above by the modulus of concavity of $\log \tilde{\phi}_{0}$.

Throughout the computations we assume that $\Omega$ has smooth boundary and is uniformly convex (so the principal curvatures of $\partial \Omega$ have a positive lower bound), and that $V$ is smooth. The results for the general case of convex $\Omega$ and semiconvex $V$ follow by using a straightforward approximation argument (see for example [HP] and [He, Thm. 2.3.17]).

The paper is arranged as follows: In Section 2 we prove a general modulus of continuity estimate for solutions of Neumann heat equations with drift. In Section 3 we use this to prove Theorem 1.3, assuming the result of Theorem 1.5. The proof 
of the modulus of continuity estimate provides a model for the more involved logconcavity estimate, which we prove in Section 4. Finally, in Section 5 we discuss some examples and extensions of our results.

\section{Modulus of CONTINUity FOR HEAT EQUATION WITH DRIFT}

We prove here a generalization of [AC2, Theorem 4.1], which controls the modulus of continuity of solutions of a Neumann heat equation with drift, in terms of the modulus of contraction of the drift velocity:

Theorem 2.1. Let $\Omega$ be a strictly convex domain of diameter $D$ with smooth boundary in $\mathbb{R}^{n}$, and $X$ a time-dependent vector field on $\Omega$. Suppose $v: \Omega \times \mathbb{R}_{+} \rightarrow \mathbb{R}$ is a smooth solution of the equation

$$
\begin{aligned}
\frac{\partial v}{\partial t} & =\Delta v+X \cdot \nabla v \quad \text { in } \Omega \times \mathbb{R}_{+} ; \\
D_{\nu} v & =0 \quad \text { in } \partial \Omega \times \mathbb{R}_{+} .
\end{aligned}
$$

Suppose that

1. $X(., t)$ has modulus of contraction $\omega(., t)$ for each $t \geq 0$, where $\omega:[0, D / 2] \times$ $\mathbb{R}_{+} \rightarrow \mathbb{R}$ is smooth;

2. $v(., 0)$ has modulus of continuity $\varphi_{0}$, where $\varphi_{0}:[0, D / 2] \rightarrow \mathbb{R}$ is smooth with $\varphi_{0}(0)=0$ and $\varphi_{0}^{\prime}(z)>0$ for $0 \leq z \leq D / 2$;

3. $\varphi:[0, D / 2] \times \mathbb{R}_{+} \rightarrow \mathbb{R}$ satisfies

(i) $\varphi(z, 0)=\varphi_{0}(z)$ for each $z \in[0, D / 2]$;

(ii) $\frac{\partial \varphi}{\partial t} \geq \varphi^{\prime \prime}+\omega \varphi^{\prime}$ on $[0, D / 2] \times \mathbb{R}_{+}$;

(iii) $\varphi^{\prime}>0$ on $[0, D / 2] \times \mathbb{R}_{+}$;

(iv) $\varphi(0, t) \geq 0$ for each $t \geq 0$.

Then $\varphi(., t)$ is a modulus of continuity for $v(., t)$ for each $t \geq 0$.

Proof. The only difference from the situation in [AC2] is the drift term in (7). For any $\varepsilon \geq 0$, define a function $Z_{\varepsilon}$ on $\bar{\Omega} \times \bar{\Omega} \times \mathbb{R}_{+}$by

$$
Z_{\varepsilon}(y, x, t)=v(y, t)-v(x, t)-2 \varphi\left(\frac{\|y-x\|}{2}, t\right)-\varepsilon \mathrm{e}^{t} .
$$

By assumption, $Z_{\varepsilon}(x, y, 0) \leq-\varepsilon$ for every $x \neq y$ in $\Omega$, and $Z_{\varepsilon}(x, x, t) \leq-\varepsilon$ for every $x \in \Omega$ and $t \geq 0$. We will prove for any $\varepsilon>0$ that $Z_{\varepsilon}$ is negative on $\Omega \times \Omega \times \mathbb{R}_{+}$. If this is not true, then there exists a first time $t_{0}>0$ and points $x \neq y \in \bar{\Omega}$ such that $Z_{\varepsilon}\left(x, y, t_{0}\right)=0$.

We consider two possibilities: If $y \in \partial \Omega$, then we have

$$
D_{\nu_{y}} Z_{\varepsilon}=\left.D_{\nu_{y}} v\right|_{(y, t)}-\varphi^{\prime} \frac{(y-x)}{\|y-x\|} \cdot \nu_{y}=-\varphi^{\prime} \frac{(y-x)}{\|y-x\|} \cdot \nu_{y}<0
$$

where $\nu_{y}$ is the outward unit normal at $y$, and we used the Neumann condition, strict convexity of $\Omega$ (which implies $\frac{y-x}{\|y-x\|} \cdot \nu_{y}>0$ ), and assumption [3ii)] This implies that $Z_{\varepsilon}\left(x, y-s \nu_{y}, t_{0}\right)>0$ for $s$ small, contradicting the fact that $Z_{\varepsilon} \leq 0$ on $\bar{\Omega} \times \bar{\Omega} \times\left[0, t_{0}\right]$. The case where $x \in \partial \Omega$ is similar.

The second possibility is that $x$ and $y$ are in the interior of $\Omega$. Then all first spatial derivatives of $Z_{\varepsilon}$ (in $x$ and $y$ ) at $\left(x, y, t_{0}\right)$ vanish, and the full $2 n \times 2 n$ matrix 
of second derivatives is nonpositive. In particular, we choose an orthonormal basis with $e_{n}=\frac{y-x}{\|y-x\|}$ and will use the inequalities

$$
\begin{aligned}
& \left.\frac{\partial^{2}}{\partial s^{2}} Z_{\varepsilon}\left(x+s e_{n}, y-s e_{n}, t_{0}\right)\right|_{s=0} \leq 0, \quad \text { and } \\
& \left.\frac{\partial^{2}}{\partial s^{2}} Z_{\varepsilon}\left(x+s e_{i}, y+s e_{i}, t_{0}\right)\right|_{s=0} \leq 0 \text { for } i=1, \ldots, n-1 .
\end{aligned}
$$

We compute these inequalities in terms of $v$ : The vanishing of first derivatives implies that

$$
\left.\nabla v\right|_{y}=\left.\nabla v\right|_{x}=\varphi^{\prime} e_{n}
$$

Along the path $\left(x+s e_{i}, y+s e_{i}\right)$ the distance $\|y-x\|$ is constant, so for $i \neq n$,

$$
0 \geq \frac{d^{2}}{d s^{2}} Z_{\varepsilon}\left(x+s e_{i}, y+s e_{i}, t_{0}\right)=\left.D_{i} D_{i} v\right|_{y}-\left.D_{i} D_{i} v\right|_{x} .
$$

Along $\left(x+s e_{n}, y-s e_{n}\right)$ we have $\frac{d}{d s}\|y-x\|=-2$ and $\frac{d^{2}}{d s^{2}}\|y-x\|=0$, so

$$
0 \geq \frac{d^{2}}{d s^{2}} Z_{\varepsilon}\left(x+s e_{n}, y-s e_{n}, t_{0}\right)=\left.D_{n} D_{n} v\right|_{y}-\left.D_{n} D_{n} v\right|_{x}-2 \varphi^{\prime \prime} .
$$

Summing the first inequality over $i=1, \ldots, n-1$ and adding the last gives

$$
0 \geq\left.\Delta v\right|_{y}-\left.\Delta v\right|_{x}-2 \varphi^{\prime \prime}
$$

Finally we compute the time derivative of $Z_{\varepsilon}$ at $\left(x, y, t_{0}\right)$ :

$$
\begin{aligned}
\frac{\partial}{\partial t} Z_{\varepsilon}= & \left.\Delta v\right|_{y}+\left.\left.X\right|_{y} \cdot \nabla v\right|_{y} \\
& -\left.\Delta v\right|_{x}-\left.\left.X\right|_{x} \cdot \nabla v\right|_{x}-2 \varphi_{t}-\varepsilon \mathrm{e}^{t} \\
\leq & 2 \varphi^{\prime \prime}+\varphi^{\prime}\left(\left.X\right|_{y}-\left.X\right|_{x}\right) \cdot \frac{y-x}{\|y-x\|}-2 \varphi_{t}-\varepsilon \mathrm{e}^{t} \\
< & 2 \varphi^{\prime \prime}+2 \omega \varphi^{\prime}-2 \varphi_{t} \\
\leq & 0
\end{aligned}
$$

where we used the modulus of contraction of $X$, and the nonnegativity of $\varphi^{\prime}$, as well as the differential inequality for $\varphi$ assumed in the theorem. But this strict inequality is impossible since this is the first time where $Z_{\varepsilon} \geq 0$. This contradiction proves that $Z_{\varepsilon}<0$ for every $\varepsilon>0$, and therefore $Z_{0} \leq 0$, which is the claim of the theorem.

\section{IMPROVED LOG-CONCAVITY IMPLIES THE GAP CONJECTURE}

We will deduce Theorem 1.3 by applying Theorem 2.1 in the case where $v$ is a ratio of solutions to the parabolic equation corresponding to the operator $\Delta+V$. We control the modulus of continuity of such a ratio assuming a modulus of concavity for the logarithm of the denominator:

Proposition 3.1. Let $u_{1}$ and $u_{0}$ be two smooth solutions to the parabolic Schrödinger equation

$$
\begin{aligned}
\frac{\partial u}{\partial t} & =\Delta u-V u \quad \text { on } \Omega \times \mathbb{R}_{+} \\
u & =0 \quad \text { on } \partial \Omega \times \mathbb{R}_{+},
\end{aligned}
$$


on a bounded strictly convex domain $\Omega$ with smooth boundary in $\mathbb{R}^{n}$, with $u_{0}$ positive on the interior of $\Omega$. Let $v(x, t)=\frac{u_{1}(x, t)}{u_{0}(x, t)}$. Then $v$ is smooth on $\Omega \times \mathbb{R}_{+}$and satisfies the following Neumann heat equation with drift:

$$
\begin{aligned}
\frac{\partial v}{\partial t}-\Delta v-2 \nabla \log u_{0} \cdot \nabla v & =0 & & \text { on } \Omega \times[0, \infty) ; \\
D_{\nu} v & =0 & & \text { on } \partial \Omega \times[0, \infty) .
\end{aligned}
$$

Proof. The argument is essentially that of [Y3, Lemma 1.1] and SWYY, Appendix A]: Both $u_{0}$ and $u_{1}$ are smooth on $\bar{\Omega} \times[0, \infty)$, and $u_{0}$ has negative derivative in the normal direction $\nu$. It follows that $v$ extends to $\bar{\Omega}$ as a smooth function (see SWYY, Section 6]). We compute directly:

$$
\begin{aligned}
\frac{\partial v}{\partial t} & =\frac{\partial}{\partial t}\left(\frac{u_{1}}{u_{0}}\right) \\
& =\frac{\Delta u_{1}-V u_{1}-v\left(\Delta u_{0}-V u_{0}\right)}{u_{0}} \\
& =\Delta v+2 \nabla \log u_{0} \cdot \nabla v .
\end{aligned}
$$

At any point in $\partial \Omega$ we have $\frac{\partial v}{\partial t}$ and $\Delta v$ bounded, $\nabla u_{0}=-c \nu$ with $c>0$, and $u_{0}=0$, so it follows from (10) that $D_{\nu} v=0$.

We now establish the fundamental gap conjecture, assuming Theorem 1.5

Proposition 3.2. Inequality (6) implies Theorem 1.3 .

Proof. We use the strategy discussed in the introduction to deduce the eigenvalue bound from an exponentially decaying modulus of continuity bound, which we deduce from Theorem 2.1 and Proposition 3.1

Suppose that $u_{1}$ is any smooth solution of (8), and let $u_{0}$ be the solution of (8) with initial data $\phi_{0}$. Let $v=\frac{u_{1}}{u_{0}}$ be the corresponding solution of (9). The drift velocity $X=2 \nabla \log u_{0}$ in equation (9) has modulus of contraction $2\left(\log \tilde{\phi}_{0}\right)^{\prime}$, by the estimate (6).

Roughly speaking, the strategy is to apply Theorem 2.1 with $\varphi=C \frac{\mathrm{e}^{-\mu_{1} t} \tilde{\phi}_{1}}{\mathrm{e}^{-\mu_{0} t} \tilde{\phi}_{0}}$ (that is, a ratio of particular solutions for the corresponding one-dimensional heat equation) and $\omega=2\left(\log \tilde{\phi}_{0}\right)^{\prime}$. Condition 2 of Theorem 2.1 holds for sufficiently large $C$, since $v(., 0)$ is smooth, and so has bounded gradient and satisfies $\mid v(y, 0)-$ $v(x, 0) \mid \leq K\|y-x\|$ for some large $K$, while $\frac{\tilde{\phi}_{1}}{\tilde{\phi}_{0}}$ is positive on $(0, D / 2)$ and has positive gradient at $z=0$, so is bounded below by $c z$ for some small $c>0$. The conditions [i] (iv) of Theorem 2.1 also hold, except that $\varphi^{\prime}(D / 2, t)=0$ and $\omega$ is not smooth at $D / 2$. We handle these complications below by making slight adjustments to $\varphi$ and $\omega$.

For small $\varepsilon>0$ we construct a smooth function $\omega_{\varepsilon} \geq \omega$ and corresponding functions $\varphi_{\varepsilon, \tilde{\varepsilon}}$ as follows: We first replace the Dirichlet eigenfunction $\tilde{\phi}_{0}$ by a Robin eigenfunction $\tilde{\phi}_{0, \varepsilon}$ with eigenvalue $\mu_{0, \varepsilon}<\mu_{0}$ defined by

$$
\begin{gathered}
\left(\tilde{\phi}_{0, \varepsilon}\right)^{\prime \prime}-\tilde{V} \tilde{\phi}_{0, \varepsilon}+\mu_{0, \varepsilon} \tilde{\phi}_{0, \varepsilon}=0 \quad \text { on }[0, D / 2] \\
\tilde{\phi}_{0, \varepsilon}(D / 2)=\varepsilon ; \quad \tilde{\phi}_{0, \varepsilon}^{\prime}(D / 2)=-1 ; \\
\tilde{\phi}_{0, \varepsilon}^{\prime}(0)=0 ; \quad \tilde{\phi}_{0, \varepsilon}>0 \quad \text { on }[0, D / 2] .
\end{gathered}
$$


We also replace $\tilde{\phi}_{1}$ by $\tilde{\phi}_{1, \tilde{\varepsilon}}$ with eigenvalue $\mu_{1, \tilde{\varepsilon}}$ defined by

$$
\begin{gathered}
\left(\tilde{\phi}_{1, \tilde{\varepsilon}}\right)^{\prime \prime}-\tilde{V} \tilde{\phi}_{1, \tilde{\varepsilon}}+\mu_{1, \tilde{\varepsilon}} \tilde{\phi}_{1, \tilde{\varepsilon}}=0 \quad \text { on }[0, D / 2] ; \\
\tilde{\phi}_{1, \tilde{\varepsilon}}(D / 2)=\tilde{\varepsilon} ; \quad \tilde{\phi}_{1, \tilde{\varepsilon}}^{\prime}(D / 2)=-1 ; \\
\tilde{\phi}_{1, \tilde{\varepsilon}}(0)=0 ; \quad \tilde{\phi}_{1, \tilde{\varepsilon}}>0 \quad \text { on }(0, D / 2] .
\end{gathered}
$$

These may be constructed using the Prüfer transformation: If $\phi^{\prime \prime}=(\tilde{V}-\mu) \phi$, then $q=\arctan \left(\phi^{\prime} / \phi\right)$ satisfies a first-order ODE. Inverting this process, we let $q\left(z, q_{0}, \mu\right)$ be the solution of

$$
\begin{aligned}
\frac{\partial q}{\partial z}-(\tilde{V}-\mu) \cos ^{2} q+\sin ^{2} q & =0, \quad\|z\| \leq D / 2, \\
q\left(0, q_{0}, \mu\right) & =q_{0} .
\end{aligned}
$$

The ODE comparison theorem implies that $q$ is strictly increasing in $q_{0}$ for all $z$, and strictly decreasing in $\mu$ for $z>0$. The choice $\mu=\mu_{0}$ and $q_{0}=0$ corresponds to $\tilde{\phi}_{0}$, and so $q\left(D / 2,0, \mu_{0}\right)=-\pi / 2$ and $q\left(z, 0, \mu_{0}\right) \in(-\pi / 2, \pi / 2)$ for $0<z<D / 2$. Similarly, the choice $\mu=\mu_{1}$ and $q_{0}=\pi / 2$ corresponds to $\tilde{\phi}_{1}$, and so $q\left(D / 2, \pi / 2, \mu_{1}\right)=-\pi / 2$ and $q\left(z, \pi / 2, \mu_{1}\right) \in(-\pi / 2, \pi / 2)$ for $0<z<D / 2$.

Since $q(D / 2,0, \mu)$ is strictly decreasing in $\mu$ and $q\left(D / 2,0, \mu_{0}\right)=-\pi / 2$, for small $\varepsilon>0$ there exists a unique $\mu_{0, \varepsilon}<\mu_{0}$ such that $q\left(D / 2,0, \mu_{0, \varepsilon}\right)=\arctan \varepsilon-\pi / 2$ and $q\left(z, 0, \mu_{0, \varepsilon}\right) \in(-\pi / 2, \pi / 2)$ for $0<z<D / 2$. The corresponding eigenfunction $\tilde{\phi}_{0, \varepsilon}(z)=\varepsilon \exp \left(-\int_{z}^{D / 2} \tan q\left(s, 0, \mu_{0, \varepsilon}\right) d s\right)$ satisfies conditions (11). We have $\left(\log \tilde{\phi}_{0, \varepsilon}\right)^{\prime}(z)=\tan q\left(z, 0, \mu_{0, \varepsilon}\right)>\tan q\left(z, 0, \mu_{0}\right)=\left(\log \tilde{\phi}_{0}\right)^{\prime}(z)$ for $z \in(0, D / 2]$, since $\mu$ was decreased. Thus $\omega_{\varepsilon}:=2\left(\log \tilde{\phi}_{0, \varepsilon}\right)^{\prime}$ is smooth and $\omega_{\varepsilon} \geq \omega$, so $\omega_{\varepsilon}$ is a modulus of contraction for $X$.

The eigenfunction $\tilde{\phi}_{1, \tilde{\varepsilon}}$ satisfying (12) is similarly produced by decreasing $\mu$ below $\mu_{1}$ with $q_{0}=\pi / 2$.

Observe that $\arctan \left(\log \tilde{\phi}_{1, \varepsilon}\right)^{\prime}(z)=q\left(z, \pi / 2, \mu_{1, \varepsilon}\right)$ satisfies the same ODE (13) as $\left(\log \tilde{\phi}_{0, \varepsilon}\right)^{\prime}(z)=q\left(z, 0, \mu_{0, \varepsilon}\right)$ with larger $\mu$, and they are equal at $z=D / 2$. By ODE comparison, $\left(\log \tilde{\phi}_{1, \varepsilon}\right)^{\prime}(z)>\left(\log \tilde{\phi}_{0, \varepsilon}\right)^{\prime}(z)$ for $0 \leq z<D / 2$, and hence if $\tilde{\varepsilon}>\varepsilon>0$, then $\left(\log \tilde{\phi}_{1, \tilde{\varepsilon}}\right)^{\prime}(z)>\left(\log \tilde{\phi}_{0, \varepsilon}\right)^{\prime}(z)$ for $0 \leq z \leq D / 2$.

For any $\tilde{\varepsilon}>\varepsilon>0$ we define a smooth function $\varphi_{\varepsilon, \tilde{\varepsilon}}$ by

$$
\varphi_{\varepsilon, \tilde{\varepsilon}}=\frac{\mathrm{e}^{-\mu_{1, \tilde{\varepsilon} t} t} \tilde{\phi}_{1, \tilde{\varepsilon}}}{\mathrm{e}^{-\mu_{0, \varepsilon} t} \tilde{\phi}_{0, \varepsilon}}
$$

Since $\varphi_{\varepsilon, \tilde{\varepsilon}}$ is a ratio of solutions of the heat equation, we have

$$
\frac{\partial}{\partial t} \varphi_{\varepsilon, \tilde{\varepsilon}}=\varphi_{\varepsilon, \tilde{\varepsilon}}^{\prime \prime}+2\left(\log \tilde{\phi}_{0, \varepsilon}\right)^{\prime} \varphi_{\varepsilon, \tilde{\varepsilon}}^{\prime}=\varphi_{\varepsilon, \tilde{\varepsilon}}^{\prime \prime}+\omega_{\varepsilon} \varphi_{\varepsilon, \tilde{\varepsilon}}^{\prime}
$$

The monotonicity requirement [iii) is satisfied, since

$$
\varphi_{\varepsilon, \tilde{\varepsilon}}^{\prime}=\varphi_{\varepsilon, \tilde{\varepsilon}}\left(\left(\log \tilde{\phi}_{1, \tilde{\varepsilon}}\right)^{\prime}-\left(\log \tilde{\phi}_{0, \varepsilon}\right)^{\prime}\right)>0 \quad \text { on }[0, D / 2] .
$$

Hence Theorem 2.1 applies to prove that $C \varphi_{\varepsilon, \tilde{\varepsilon}}(., t)$ is a modulus of continuity for $v$ for $t>0$ if it is so at $t=0$. Sending $\tilde{\varepsilon}$ to $\varepsilon$ and then sending $\varepsilon$ to zero, we have that $\varphi_{\varepsilon, \tilde{\varepsilon}}$ converges uniformly to $\varphi$, so the same conclusion holds for $\varphi$. In particular, for sufficiently large $C$ we have

$$
\operatorname{osc} v(., t) \leq 2 C \sup \{\varphi(z, t): z \in[0, D / 2]\}=2 C \mathrm{e}^{-\left(\mu_{1}-\mu_{0}\right) t} .
$$


Applying this with $u_{1}=\phi_{1} \mathrm{e}^{-\lambda_{1} t}$ gives $v=\mathrm{e}^{-\left(\lambda_{1}-\lambda_{0}\right) t} \frac{\phi_{1}}{\phi_{0}}$, and hence

$$
\mathrm{e}^{-\left(\lambda_{1}-\lambda_{0}\right) t} \operatorname{osc} \frac{\phi_{1}}{\phi_{0}} \leq 2 C \mathrm{e}^{-\left(\mu_{1}-\mu_{0}\right) t}
$$

for all $t \geq 0$, which implies $\lambda_{1}-\lambda_{0} \geq \mu_{1}-\mu_{0}$. This proves Theorem 1.3 ,

\section{ImPROVED LOG-CONCAVITY OF THE GROUND STATE}

In this section we prove the log-concavity result, Theorem 1.5. We will deduce this from the following statement about log-concavity for arbitrary positive solutions of the parabolic equation (8):

Theorem 4.1. Let $\Omega$ be a uniformly convex open domain with diameter $D$ in $\mathbb{R}^{n}$, and assume that $\tilde{V}^{\prime}$ is a modulus of convexity for the potential $V$ on $\Omega$. Let $u_{0}$ be a smooth function on $\bar{\Omega}$ which is positive on $\Omega$ and has $u_{0}=0$ and $\nabla u_{0} \neq 0$ on $\partial \Omega$, and suppose $\psi_{0}:[0, D / 2] \rightarrow \mathbb{R}$ is a Lipschitz continuous modulus of concavity for $\log u_{0}$. Let $u: \Omega \times \mathbb{R}_{+} \rightarrow \mathbb{R}$ be the solution of the Dirichlet problem (8) with initial data $u_{0}$, and let $\psi \in C^{0}\left([0, D / 2] \times \mathbb{R}_{+}\right) \cap C^{\infty}([0, D / 2] \times(0, \infty))$ be a solution of

$$
\left.\begin{array}{c}
\frac{\partial \psi}{\partial t} \geq \psi^{\prime \prime}+2 \psi \psi^{\prime}-\tilde{V}^{\prime} \quad \text { on }[0, D / 2] \times \mathbb{R}_{+}, \\
\psi(., 0)=\psi_{0}(.), \\
\psi(0, t)=0 .
\end{array}\right\}
$$

Then $\psi(., t)$ is a modulus of concavity for $\log u(., t)$ for each $t \geq 0$.

Note that we impose no boundary condition on $\psi$ at $(D / 2, t)$.

Proof. The statement we must prove is the following:

$$
Z(x, y, t):=\left(\left.\nabla \log u\right|_{(y, t)}-\left.\nabla \log u\right|_{(x, t)}\right) \cdot \frac{y-x}{\|y-x\|}-2 \psi\left(\frac{\|y-x\|}{2}, t\right) \leq 0
$$

for all $x \neq y \in \Omega$ and $t \geq 0$. By assumption this holds for $t=0$. We will prove this by showing negativity of a function $Z_{\varepsilon}$ on $\hat{\Omega} \times[0, T]$ for any $\varepsilon>0$ and $T \in(0, \infty)$, where

$$
\hat{\Omega}=(\Omega \times \Omega) \backslash\{y=x\} \subset \mathbb{R}^{2 n}
$$

and $Z_{\varepsilon}$ is defined by

$$
\begin{aligned}
Z_{\varepsilon}(x, y, t) & =Z(x, y, t)-\varepsilon \mathrm{e}^{C t} \\
& =\left(\left.\nabla \log u\right|_{y}-\left.\nabla \log u\right|_{x}\right) \cdot \frac{y-x}{\|y-x\|}-2 \psi\left(\frac{\|y-x\|}{2}, t\right)-\varepsilon \mathrm{e}^{C t},
\end{aligned}
$$

for some suitably large $C$ to be chosen (independent of $\varepsilon$ ), where we have suppressed the $t$-dependence of $u$. By the Hopf boundary point lemma, $D_{\nu} u(x, t)<0$ for every $x \in \partial \Omega$ and every $t \geq 0$. The following lemmas control the behaviour of $Z$ near the boundary:

Lemma 4.2. Let $u$ be as in Theorem 4.1, and $T<\infty$. Then there exists $r_{1}>0$ such that $\left.D^{2} \log u\right|_{(x, t)}<0$ whenever $d(x, \partial \Omega)<r_{1}$ and $t \in[0, T]$, and $N \in \mathbb{R}$ such that $\left.D^{2} \log u\right|_{(x, t)}(v, v) \leq N\|v\|^{2}$ for all $x \in \Omega$ and $t \in[0, T]$. 
Proof. Let $\alpha=\inf _{\partial \Omega \times[0, T]}\|D u\|>0$, and choose a constant $K$ such that $\left|D^{2} u(v, v)\right|$ $\leq K\|v\|^{2}$ at every point in $\bar{\Omega} \times[0, T]$ and $v \in \mathbb{R}^{n}$. If $x_{0} \in \partial \Omega$, then $\left.D u\right|_{x_{0}}=$ - $\|\left. D u\right|_{x_{0}} \mid \nu \nu\left(x_{0}\right)$, and $\left.D^{2} u\right|_{x_{0}}(v, v)=\left.h(v, v) D u\right|_{x_{0}}\left(\nu\left(x_{0}\right)\right)$ for $v$ tangent to $\partial \Omega$, where $h$ is the second fundamental form of $\partial \Omega$ at $x_{0}$ (see, for example, GT, §14.6]). Uniform convexity implies that $h(v, v) \geq \kappa\|v\|^{2}$ for some $\kappa>0$. The gradient direction $e=\frac{D u}{\|D u\|}$ is smooth near $x_{0}$, as is the projection $\pi^{\perp}: w \mapsto(w \cdot e) e$ and the orthogonal projection $\pi=\mathrm{Id}-\pi^{\perp}$. At $x_{0}$ we have $D^{2} u(\pi w, \pi w) \leq-\alpha \kappa\|\pi w\|^{2}$. Therefore there exists $r_{0}>0$ depending on $\alpha$ and $K$ such that for $x \in B_{r_{0}}\left(x_{0}\right) \cap \Omega$ and $t \in[0, T]$ we have

$$
\begin{aligned}
\left.D^{2} u\right|_{x}(\pi w, \pi w) & \leq-\frac{\alpha \kappa}{2}\|\pi w\|^{2} \quad \text { for any } w \in \mathbb{R}^{n} ; \\
\left\|\left.D u\right|_{x}\right\| & \geq \frac{1}{2}\left\|\left.D u\right|_{x_{0}}\right\| ; \\
0<u(x) & \leq 2\left\|\left.D u\right|_{x_{0}}\right\|\left\|x-x_{0}\right\| .
\end{aligned}
$$

Therefore in this set and for any $w$, we have

$$
\begin{aligned}
D^{2} u(w, w) & =D^{2} u\left(\pi w+\pi^{\perp} w, \pi w+\pi^{\perp} w\right) \\
& \leq-\frac{1}{2} \alpha \kappa\|\pi w\|^{2}+2 K\|\pi w\|\left\|\pi^{\perp} w\right\|+K\left\|\pi^{\perp} w\right\|^{2} \\
& \leq-\frac{1}{4} \alpha \kappa\|\pi w\|^{2}+\left(K+\frac{4 K^{2}}{\alpha \kappa}\right)\left\|\pi^{\perp} w\right\|^{2} .
\end{aligned}
$$

By definition of $\pi$ we have $\left(D_{w} u\right)^{2}=\|D u\|^{2}\left\|\pi^{\perp} w\right\|^{2} \geq \frac{\alpha\left\|\left.D u\right|_{x_{0}}\right\|}{4}\left\|\pi^{\perp} w\right\|^{2}$, and also $u \leq 2\left\|\left.D u\right|_{x_{0}}\right\|\left\|x-x_{0}\right\|$, so

$$
\begin{aligned}
\left.D^{2} \log u\right|_{x}(w, w) & =\frac{1}{u}\left(D^{2} u(w, w)-\frac{\left(D_{w} u\right)^{2}}{u}\right) \\
& \leq \frac{1}{u}\left(-\frac{1}{4} \alpha \kappa\|\pi w\|^{2}+\left(K+\frac{4 K^{2}}{\alpha \kappa}\right)\left\|\pi^{\perp} w\right\|^{2}\right. \\
& <0
\end{aligned}
$$

provided $\left\|x-x_{0}\right\|<r_{1}=\min \left\{r_{0}, \frac{\alpha^{2} \kappa}{8\left(K \alpha \kappa+4 K^{2}\right)}\right\}$. The lemma holds with $N=$ $\max \left\{0, \sup \left\{D^{2} \log u(z, t)(v, v):\|v\|=1, t \in[0, T], d(z, \partial \Omega) \geq r_{1}\right\}\right\}$, since $\{z \in$ $\left.\Omega: d(z, \partial \Omega) \geq r_{1}\right\}$ is compact.

Lemma 4.3. Let $u$ be as in Theorem 4.1, and let $\psi$ be continuous on $[0, D / 2] \times \mathbb{R}_{+}$ and locally Lipschitz in the first argument, with $\psi(0, t)=0$ for each $t$. Then for any $T<\infty$ and $\beta>0$ there exists an open set $U_{\beta, T} \subset \mathbb{R}^{2 n}$ containing $\partial \hat{\Omega}$ such that $Z(x, y, t)<\beta$ for all $t \in[0, T]$ and $(x, y) \in U_{\beta, T} \cap \hat{\Omega}$.

Proof. Since $\psi$ is Lipschitz in the first argument there exists $P$ such that $|\psi(z, t)| \leq$ $P z$ for all $z \in[0, D / 2]$ and $t \in[0, T]$.

We construct $U_{\beta, T}$ of the form $\bigcup_{\left(x_{0}, y_{0}\right) \in \partial \hat{\Omega}} B_{r\left(x_{0}, y_{0}\right)}\left(x_{0}, y_{0}\right)$. To do this we must find $r\left(x_{0}, y_{0}\right)>0$ for any $\left(x_{0}, y_{0}\right) \in \partial \hat{\Omega}$ such that $Z<\beta$ for $(x, y) \in B_{r\left(x_{0}, y_{0}\right)}\left(x_{0}, y_{0}\right)$. We consider several cases for $\left(x_{0}, y_{0}\right)$ : 
Case 1: $x_{0}=y_{0} \in \Omega$. We have by Lemma 4.2 ,

$$
\begin{aligned}
Z(x, y, t) & =\left(\left.\nabla \log u\right|_{(y, t)}-\left.\nabla \log u\right|_{(x, t)}\right) \cdot \frac{y-x}{\|y-x\|}-2 \psi\left(\frac{\|y-x\|}{2}, t\right) \\
& =\left.\frac{1}{\|y-x\|} \int_{0}^{1} D^{2} \log u\right|_{(1-s) x+s y}(y-x, y-x) d s-2 \psi\left(\frac{\|y-x\|}{2}, t\right) \\
& \leq(N+P)\|y-x\|,
\end{aligned}
$$

so $Z<\beta$ provided $(x, y) \in B_{r}\left(x_{0}, x_{0}\right)$ with $r<\frac{\beta}{2(N+P)}$.

Case 2: $x_{0} \in \partial \Omega, y_{0} \in \Omega$. In this case $u\left(y_{0}\right)>0$, so there exists $r_{2}>0$ and $L>0$ such that $\left\|\left.D \log u\right|_{y}\right\| \leq L$ for $\left\|y-y_{0}\right\|<r_{2}$. Let $\alpha_{0}=\left\|\left.D u\right|_{x_{0}}\right\|>0$ and $\gamma=-\frac{y_{0}-x_{0}}{\left\|y_{0}-x_{0}\right\|} \cdot \nu\left(x_{0}\right)$ (this is positive by strict convexity). Then $D u\left(x_{0}\right)=-\alpha_{0} \nu\left(x_{0}\right)$, so $\left.D u\right|_{x_{0}} \cdot \frac{y_{0}-x_{0}}{\left\|y_{0}-x_{0}\right\|}=\gamma \alpha_{0}$. In addition, $\left.D u\right|_{x} \cdot \frac{y-x}{\|y-x\|}$ is smooth in $x$ and $y$ near $\left(x_{0}, y_{0}\right)$. Therefore $\left.D u\right|_{x} \cdot \frac{y-x}{\|y-x\|} \geq \frac{1}{2} \gamma \alpha_{0}$ and $0<u(x) \leq 2 \alpha_{0}\left\|x-x_{0}\right\|$ for $x, y \in \Omega$ with $\max \left\{\left\|y-y_{0}\right\|,\left\|x-x_{0}\right\|\right\}<r_{3}$, for some $r_{3} \in\left(0, r_{2}\right]$. This implies that

$$
\begin{aligned}
Z(x, y, t) & =\left(\left.\nabla \log u\right|_{(y, t)}-\left.\nabla \log u\right|_{(x, t)}\right) \cdot \frac{y-x}{\|y-x\|}-2 \psi\left(\frac{\|y-x\|}{2}, t\right) \\
& \leq L-\frac{\gamma}{4\left\|x-x_{0}\right\|}+P D,
\end{aligned}
$$

where $D$ is diameter, so $Z<0$ provided $\left\|y-y_{0}\right\|<r_{3}$ and $\left\|x-x_{0}\right\|<$ $\min \left\{r_{3}, \frac{\gamma}{4(L+P D)}\right\}$.

Case 3: $x_{0} \in \Omega, y_{0} \in \partial \Omega$. This is similar to Case 2.

Case 4: $x_{0}, y_{0} \in \partial \Omega, x_{0} \neq y_{0}$. Here both $x$ and $y$ can be handled in the same way as $x$ in Case 2

Case 5: $x_{0}=y_{0} \in \partial \Omega$. Then letting $z_{s}=(1-s) x+s y$ we write as in Case 1.

$$
Z(x, y, t)=\left.\frac{1}{\|y-x\|} \int_{0}^{1} D^{2} \log u\right|_{z_{s}}(y-x, y-x) d s-2 \psi\left(\frac{\|y-x\|}{2}, t\right) .
$$

The first term is negative for $\max \left\{\left\|x-x_{0}\right\|,\left\|y-x_{0}\right\|\right\}<r_{1}$ by Lemma 4.2. so $Z(x, y, t)<\beta$ provided $\max \left\{\left\|x-x_{0}\right\|,\left\|y-x_{0}\right\|\right\}<\min \left\{r_{1}, \frac{\beta}{P}\right\}$.

Now we proceed with the proof of Theorem 4.1. Fix $T<\infty$ and $\varepsilon>0$. By assumption we have $Z_{\varepsilon}(x, y, 0) \leq-\varepsilon \mathrm{e}^{C t}<0$ for all $x \neq y$ in $\Omega$. Off the compact set $\left(\hat{\Omega} \backslash U_{\varepsilon / 2, T}\right) \times[0, T]$ we have $Z_{\varepsilon} \leq-\frac{1}{2} \varepsilon$, by Lemma 4.3. Therefore if $Z_{\varepsilon}$ is not negative on $\hat{\Omega} \times[0, T]$, then there exists a first time $t_{0}>0$ and points $(x, y) \in \hat{\Omega} \backslash U_{\varepsilon / 2, T}$ such that $Z_{\varepsilon}<0$ on $\hat{\Omega} \times\left[0, t_{0}\right)$, but $Z_{\varepsilon}\left(x, y, t_{0}\right)=0$. In particular $x$ and $y$ are in the interior of $\Omega$, and $y \neq x$. We choose an orthonormal basis $\left\{e_{i}\right\}$ for $\mathbb{R}^{n}$ with $e_{n}=\frac{y-x}{\|y-x\|}$, and note the following: The first spatial derivatives of $Z_{\varepsilon}$ in $x$ and $y$ 
vanish, so

$$
\begin{aligned}
0 & =\left.\frac{\partial}{\partial s} Z_{\varepsilon}\left(x+s e_{i}, y, t_{0}\right)\right|_{s=0}, \quad 1 \leq i<n \\
& =-\left.D_{i} D_{n} \log u\right|_{x}-\frac{\left.D_{i} \log u\right|_{y}-\left.D_{i} \log u\right|_{x}}{\|y-x\|} \\
0 & =\left.\frac{\partial}{\partial s} Z_{\varepsilon}\left(x, y+s e_{i}, t_{0}\right)\right|_{s=0}, \quad 1 \leq i<n \\
& =\left.D_{i} D_{n} \log u\right|_{y}+\frac{\left.D_{i} \log u\right|_{y}-\left.D_{i} \log u\right|_{x}}{\|y-x\|}
\end{aligned}
$$

while

$$
\begin{aligned}
& 0=\left.\frac{\partial}{\partial s} Z_{\varepsilon}\left(x+s e_{n}, y, t_{0}\right)\right|_{s=0}=-\left.D_{n} D_{n} \log u\right|_{x}+\psi^{\prime} ; \\
& 0=\left.\frac{\partial}{\partial s} Z_{\varepsilon}\left(x, y+s e_{n}, t_{0}\right)\right|_{s=0}=\left.D_{n} D_{n} \log u\right|_{y}-\psi^{\prime} .
\end{aligned}
$$

The second derivative of $Z_{\varepsilon}\left(., ., t_{0}\right)$ along any path is nonpositive at $(x, y)$. In particular, $y-x$ is constant along $\left(x+s e_{i}, y+s e_{i}\right)$ for $1 \leq i<n$, so

$$
\begin{aligned}
0 & \geq\left.\frac{\partial^{2}}{\partial s^{2}} Z_{\varepsilon}\left(x+s e_{i}, y+s e_{i}, t_{0}\right)\right|_{s=0} \\
& =\left.D_{i} D_{i} D_{n} \log u\right|_{y}-\left.D_{i} D_{i} D_{n} \log u\right|_{x}
\end{aligned}
$$

Along the path $\left(x+s e_{n}, y-s e_{n}\right)$ we have $\frac{y-x}{\|y-x\|}$ constant, $\frac{d}{d s}\|y-x\|=-2$, and $\frac{d^{2}}{d s^{2}}\|y-x\|=0$. This gives

$$
\begin{aligned}
0 & \geq\left.\frac{\partial^{2}}{\partial s^{2}} Z_{\varepsilon}\left(x+s e_{n}, y-s e_{n}, t_{0}\right)\right|_{s=0} \\
& =\left.D_{n} D_{n} D_{n} \log u\right|_{y}-\left.D_{n} D_{n} D_{n} \log u\right|_{x}-2 \psi^{\prime \prime}
\end{aligned}
$$

Finally, since $Z_{\varepsilon}(x, y, t)<Z_{\varepsilon}\left(x, y, t_{0}\right)$ for $t<t_{0}$, we have $\frac{\partial Z_{\varepsilon}}{\partial t}\left(x, y, t_{0}\right) \geq 0$. We have $\frac{\partial}{\partial t} \log u=\Delta \log u+\|D \log u\|^{2}-V$ by (8), and differentiation gives

$$
\frac{\partial}{\partial t} \nabla \log u=\Delta \nabla \log u+\sum_{k=1}^{n} 2 D_{k} \log u D_{k} \nabla \log u-\nabla V
$$


This gives the following inequality:

$$
\begin{aligned}
0 \leq & \frac{\partial}{\partial t} Z_{\varepsilon} \\
= & \left(\left.\Delta \nabla \log u\right|_{y}+\left.\sum_{k=1}^{n} 2 D_{k} \log u D_{k} \nabla \log u\right|_{y}-\left.\nabla V\right|_{y}\right. \\
& \left.-\left.\Delta \nabla \log u\right|_{x}-\left.\sum_{k=1}^{n} 2 D_{k} \log u D_{k} \nabla \log u\right|_{x}+\left.\nabla V\right|_{x}\right) \cdot \frac{y-x}{\|y-x\|} \\
& -2 \frac{\partial \psi}{\partial t}-C \varepsilon \mathrm{e}^{C t} \\
= & \left.D_{n} D_{n} D_{n} \log u\right|_{y}-\left.D_{n} D_{n} D_{n} \log u\right|_{x} \\
& +\sum_{i=1}^{n-1}\left(\left.D_{i} D_{i} D_{n} \log u\right|_{y}-\left.D_{i} D_{i} D_{n} \log u\right|_{x}\right) \\
& +2\left(\left.\left.D_{n} \log u\right|_{y} D_{n} D_{n} \log u\right|_{y}-\left.\left.D_{n} \log u\right|_{x} D_{n} D_{n} \log u\right|_{x}\right) \\
& +2 \sum_{i=1}^{n-2}\left(\left.\left.D_{i} \log u\right|_{y} D_{i} D_{n} \log u\right|_{y}-\left.\left.D_{i} \log u\right|_{x} D_{i} D_{n} \log u\right|_{x}\right) \\
& -\left(\left.\nabla V\right|_{y}-\left.\nabla V\right|_{x}\right) \cdot \frac{y-x}{\|y-x\|}-2 \frac{\partial \psi}{\partial t}-C \varepsilon \mathrm{e}^{C t} .
\end{aligned}
$$

In the first line we use the second derivative inequality (16b), and in the second we use (16a). In the third line we rewrite the second derivatives of $\log u$ using (15c) and (15d), while in the fourth we use (15a) and (15b). In the last line we use the modulus of convexity assumption (5). This yields

$$
\begin{aligned}
0 \leq & 2 \psi^{\prime \prime}+2 \psi^{\prime}\left(\left.D_{n} \log u\right|_{y}-\left.D_{n} \log u\right|_{x}\right) \\
& -\frac{2}{\|y-x\|} \sum_{i=1}^{n-1}\left|D_{i} \log u\right|_{y}-\left.\left.D_{i} \log u\right|_{x}\right|^{2}-2 \tilde{V}^{\prime}-2 \frac{\partial \psi}{\partial t}-C \varepsilon \mathrm{e}^{C t} \\
\leq & 2 \psi^{\prime \prime}+2 \psi^{\prime}\left(2 \psi+\varepsilon \mathrm{e}^{C t}\right)-2 \tilde{V}^{\prime}-2 \frac{\partial \psi}{\partial t}-C \varepsilon \mathrm{e}^{C t} \\
& <2\left(\psi^{\prime \prime}+2 \psi \psi^{\prime}-\tilde{V}^{\prime}-\frac{\partial \psi}{\partial t}\right) \leq 0 .
\end{aligned}
$$

Here the second inequality is obtained by discarding the negative term involving $D_{i} \log u$, and using $Z_{\varepsilon}=\left.D_{n} \log u\right|_{y}-\left.D_{n} \log u\right|_{x}-2 \psi-\varepsilon \mathrm{e}^{C t}=0$. The last inequality is from equation (14), and the strict inequality is produced by choosing $C>2 \sup _{[0, D / 2] \times[0, T]} \psi^{\prime}$ (this is independent of $\varepsilon$, as required). The result contradicts the assumption that $Z_{\varepsilon}$ does not remain negative. Therefore $Z_{\varepsilon}<0$ on $\hat{\Omega} \times[0, T]$ for every $\varepsilon>0$ and every $T<\infty$. It follows that $Z \leq 0$ on $\hat{\Omega} \times \mathbb{R}_{+}$, and the theorem is proved.

We next consider the long-term behaviour of solutions of (14):

Corollary 4.4. Under the conditions of Theorem 4.1, there exists a smooth function $\psi:[0, D / 2) \times \mathbb{R}_{+}$, decreasing in the second argument, such that

$$
\left(\left.\nabla \log u\right|_{(y, t)}-\nabla \log u \mid+(x, t)\right) \cdot \frac{y-x}{\|y-x\|} \leq 2 \psi\left(\frac{\|y-x\|}{2}, t\right)
$$


for all $x, y \in \Omega$ with $x \neq y$ and all $t \geq 0$, and $\lim _{t \rightarrow \infty} \psi(z, t)=\left(\log \tilde{\phi}_{0}\right)^{\prime}(z)$ for every $z \in[0, D / 2)$.

Proof. We construct a sequence of functions $\psi_{k}$ satisfying the assumptions of Theorem 4.1 and decreasing monotonically towards the (nonsmooth) function $\psi$ as $k \rightarrow$ $\infty$. These are constructed by solving the equality case of (14) with $\psi(D / 2, t)=-k$ for suitable initial data.

The first step is to construct suitable initial functions $\psi_{k, 0}$. To do this we observe that there are many stationary solutions, corresponding to solutions of the eigenvalue equation via the Prüfer transformation: Stationary solutions satisfy $0=\psi^{\prime \prime}+2 \psi \psi^{\prime}-\tilde{V}^{\prime}=\left(\psi^{\prime}+\psi^{2}-\tilde{V}\right)^{\prime}$, and hence

$$
\psi^{\prime}+\psi^{2}=\tilde{V}-\mu
$$

for some $\mu$. We consider solutions $\psi_{\mu}^{L}$ and $\psi_{k, \mu}^{R}$ of (17) satisfying $\psi_{\mu}^{L}(0)=0$ and $\psi_{k, \mu}^{R}(D / 2)=-k$. These relate to the Robin eigenfunctions $\tilde{\phi}_{0, \varepsilon}$ satisfying equation (11), which were constructed in the proof of Proposition 3.2, with $\varepsilon=1 / k$ : In particular, $\psi_{\mu_{0,1 / k}}^{L}=\psi_{k, \mu_{0,1 / k}}^{R}=\left(\log \tilde{\phi}_{0,1 / k}\right)^{\prime}$. The ODE comparison theorem applied to equation (17) implies that $\psi_{\mu}^{L}$ is strictly decreasing in $\mu$ for $0<z \leq$ $D / 2$, and $\psi_{k, \mu}^{R}$ is strictly increasing in $\mu$ for $0 \leq z<D / 2$. In particular, $\psi_{k, \mu}^{R}>$ $\left(\log \tilde{\phi}_{0,1 / k}\right)^{\prime}$ for $\mu>\mu_{0,1 / k}$ and $0 \leq z<D / 2$, and $\psi_{\mu}^{L}>\left(\log \tilde{\phi}_{0,1 / k}\right)^{\prime}$ for $\mu<\mu_{0,1 / k}$ and $0<z \leq D / 2$. ODE comparison also gives upper bounds: If $\lambda_{+}^{2} \geq \sup \tilde{V}-\mu$ and $\lambda_{-}^{2} \geq \mu-\inf \tilde{V}$, then

$$
\begin{aligned}
\psi_{\mu}^{L}(z) & \leq \lambda_{+} \tanh \left(\lambda_{+} z\right), \quad \text { and } \\
\psi_{k, \mu}^{R}(z) & \leq \frac{\lambda_{-} \tan \left(\lambda_{-}(D / 2-z)\right)-k}{1+\frac{k}{\lambda_{-}} \tan \left(\lambda_{-}(D / 2-z)\right)} \text { if } z>\frac{D}{2}-\frac{\frac{\pi}{2}+\arctan \left(\frac{k}{\lambda_{-}}\right)}{\lambda_{-}} .
\end{aligned}
$$

The upper and lower bounds imply the existence of a supersolution $\psi_{k, s}^{+}=$ $\min \left\{\psi_{\mu_{0,1 / k}-s}^{L}, \psi_{k, \mu_{0,1 / k}+s}^{R}\right\}$ for any $s \geq 0$. Comparison gives lower bounds on these for large $s$ : For $s>\max \left\{\mu_{0,1 / k}-\inf \tilde{V}, \sup \tilde{V}-\mu_{0,1 / k}\right\}$,

$$
\psi_{k, s}^{+}(z) \geq \begin{cases}\tilde{\lambda}_{+} \tanh \left(\tilde{\lambda}_{+} z\right), & 0 \leq z \leq z_{0} \\ \frac{\tilde{\lambda}_{-} \tan \left(\tilde{\lambda}_{-}(D / 2-z)\right)-k}{1+\frac{k}{\lambda_{-}} \tan \left(\tilde{\lambda}_{-}(D / 2-z)\right)}, & z_{0} \leq z \leq \frac{D}{2}\end{cases}
$$

where $\tilde{\lambda}_{+}=\sqrt{s+\inf \tilde{V}-\mu_{0,1 / k}}, \tilde{\lambda}_{-}=\sqrt{s+\mu_{0,1 / k}-\sup \tilde{V}}$, and we take $z_{0}>$ $\frac{D}{2}-\tilde{\lambda}_{-}^{-1}\left(\frac{\pi}{2}+\arctan \left(\frac{k}{\tilde{\lambda}_{-}}\right)\right)$to make the two cases equal.

Lemma 4.5. For $u_{0}$ as in Theorem 4.1, for each $k$ there exists $s(k) \geq 0$ such that $\psi_{k, s}^{+}$is a modulus of concavity for $\log u_{0}$.

Proof. By Lemma 4.2, we have for all $(y, x) \in \hat{\Omega}$,

$$
\left(\left.\nabla \log u_{0}\right|_{y}-\left.\nabla \log u_{0}\right|_{x}\right) \cdot \frac{y-x}{\|y-x\|} \leq N\|y-x\| \leq 2 \lambda_{1} \tanh \left(\frac{\lambda_{1}\|y-x\|}{2}\right),
$$


where $\lambda_{1}$ is such that $N D \leq 2 \lambda_{1} \tanh \left(\lambda_{1} D / 2\right)$. Now apply Lemma 4.3 with $\psi(z)=$ $-\frac{6 k z}{D}$ and $\beta=k$ to find an open set $U \subset \mathbb{R}^{2}$ containing $\partial \hat{\Omega}$ such that

$$
\left(\left.\nabla \log u_{0}\right|_{y}-\left.\nabla \log u_{0}\right|_{x}\right) \cdot \frac{y-x}{\|y-x\|} \leq-\frac{6 k\|y-x\|}{D}+k
$$

for $(x, y) \in \hat{\Omega} \cap U$. In particular, there exists $\delta>0$ such that $U$ contains all points $(x, y) \in \hat{\Omega}$ with $\|y-x\| \geq D-\delta$. Decreasing $\delta$ so that $\delta<D / 3$ if necessary, we have that for all $(x, y) \in \hat{\Omega}$ with $\|y-x\| \geq D-\delta$,

$$
\begin{aligned}
\left(\left.\nabla \log u_{0}\right|_{y}-\left.\nabla \log u_{0}\right|_{x}\right) \cdot \frac{y-x}{\|y-x\|} & \leq-\frac{6 k(D-\delta)}{D}+k \\
& \leq-2 k \\
& \leq 2 \frac{\lambda_{2} \tan \left(\lambda_{2}\left(\frac{D-\|y-x\|}{2}\right)\right)-k}{1+\frac{k}{\lambda_{2}} \tan \left(\lambda_{2}\left(\frac{D-\|y-x\|}{2}\right)\right)}
\end{aligned}
$$

for any $\lambda_{2}>0$, provided $\frac{D-\|y-x\|}{2}<\frac{\frac{\pi}{2}+\arctan \left(\frac{k}{\lambda_{2}}\right)}{\lambda_{2}}$. We choose $\lambda_{2}$ so large that $\frac{\pi}{2}+\arctan \left(\frac{k}{\lambda_{2}}\right)<\delta \lambda_{2}$. Then by (18) we have for all $(x, y) \in \hat{\Omega}$,

$$
\left(\left.\nabla \log u_{0}\right|_{y}-\left.\nabla \log u_{0}\right|_{x}\right) \cdot \frac{y-x}{\|y-x\|} \leq \psi_{k, s}^{+}\left(\frac{\|y-x\|}{2}\right)
$$

provided $s \geq \max \left\{\lambda_{1}^{2}+\mu_{0,1 / k}-\inf \tilde{V}, \lambda_{2}^{2}+\sup \tilde{V}-\mu_{0,1 / k}\right\}$.

Now we proceed to construct the solutions $\psi_{k}$ : We define

$$
s(k)=\inf \left\{s \geq 0: \psi_{k, s}^{+} \text {is a modulus of concavity for } \log u_{0}\right\} .
$$

Then we choose the initial data as follows:

$$
\psi_{k, 0}=\min \left\{\psi_{j, s(j)}^{+}: 1 \leq j \leq k\right\} .
$$

Note that $\psi_{k, 0}$ is pointwise nonincreasing in $k$. Now define $\psi_{k}(z, t)$ by

$$
\left.\begin{array}{c}
\frac{\partial \psi_{k}}{\partial t}=\psi_{k}^{\prime \prime}+2 \psi_{k} \psi_{k}^{\prime}-\tilde{V}^{\prime} \quad \text { on }[0, D / 2] \times(0, \infty), \\
\psi_{k}(., 0)=\psi_{k, 0}(.), \\
\psi_{k}(0, t)=0, \\
\psi_{k}(D / 2, t)=-k .
\end{array}\right\}
$$

The solution $\psi_{k}$ remains between the supersolution $\psi_{k, 0}$ and the subsolution $\left(\log \tilde{\phi}_{0,1 / k}\right)^{\prime}$ and is decreasing in $t$ and in $k$. The inequality $\frac{\partial \psi_{k}}{\partial t} \leq 0$ implies bounds on $\psi_{k}^{\prime}$ (since the barriers bound $\psi_{k}^{\prime}$ at the endpoints), and it follows that $\psi_{k}$ exists and is Lipschitz in the first argument and continuous on $[0, D / 2] \times \mathbb{R}_{+}$, and smooth on $[0, D / 2] \times(0, \infty)$. By the strong maximum principle, $\psi_{k}(z, t)$ is strictly decreasing in $t$ on $(0, D / 2) \times \mathbb{R}_{+}$unless $\psi_{k}=\left(\log \tilde{\phi}_{0,1 / k}\right)^{\prime}$. It follows that for each $k, \psi_{k}(., t)$ converges in $C^{\infty}$ to $\left(\log \tilde{\phi}_{0,1 / k}\right)^{\prime}$ as $t \rightarrow \infty$. By Theorem $4.1 \psi_{k, t}$ is a modulus of concavity for $\log u(., t)$ for each $t>0$ and each $k \in \mathbb{N}$, and therefore $\psi(., t)=\lim _{k \rightarrow \infty} \psi_{k}(., t)=\min _{k \in \mathbb{N}} \psi_{k}(., t)$ is also. Since $\psi_{k}$ is bounded independently of $k$ in $C^{q}$ for every $q$ on compact subsets of $[0, D / 2) \times(0, \infty), \psi$ is a smooth 
solution of

$$
\left.\begin{array}{c}
\frac{\partial \psi}{\partial t}=\psi^{\prime \prime}+2 \psi \psi^{\prime}-\tilde{V}^{\prime} \quad \text { on }[0, D / 2) \times(0, \infty), \\
\psi(0, t)=0, \\
\lim _{z \rightarrow D / 2} \psi(z, t)=-\infty,
\end{array}\right\}
$$

and $\psi(., t)$ converges in $C^{\infty}$ to $\left(\log \tilde{\phi}_{0}\right)^{\prime}$ as $t \rightarrow \infty$ on compact subsets of $[0, D / 2)$. This completes the proof.

Proof of Theorem 1.5. Theorem 1.5 follows by applying Corollary 4.4 to the particular solution $u(x, t)=\phi_{0}(x) \mathrm{e}^{-\lambda_{1} t}$ of (8). In this case, $\nabla \log u$ is independent of $t$. Therefore $\psi(., t)$ is a modulus of concavity for $\log u_{0}$ for every $t$, and hence $\left(\log \tilde{\phi}_{0}\right)^{\prime}=\inf _{t \geq 0} \psi(., t)$ is also.

\section{EXAMPLES AND EXTENSIONS}

When $V$ is convex, Theorem 1.3 gives Corollary 1.4, since then 0 is a modulus of convexity for $V$, so we can choose $\tilde{V}=0$ and the first two eigenfunctions are $\tilde{\phi}_{0}(z)=\cos \left(\frac{\pi z}{D}\right)$ with $\mu_{0}=\frac{\pi^{2}}{D^{2}}$, and $\tilde{\phi}_{1}(z)=\cos \left(\frac{2 \pi z}{D}\right)$ with $\mu_{1}=\frac{4 \pi^{2}}{D^{2}}$, and hence $\lambda_{1}-\lambda_{0} \geq \mu_{1}-\mu_{0}=\frac{3 \pi^{2}}{D^{3}}$.

A positive lower bound on the Hessian of the potential implies stronger estimates on the gap since a particular modulus of convexity follows:

$$
\begin{aligned}
\left(\left.\nabla V\right|_{y}-\left.\nabla V\right|_{x}\right) \cdot \frac{y-x}{\|y-x\|} & =\left.\frac{1}{\|y-x\|} \int_{0}^{1} D^{2} V\right|_{(1-s) x+s y}(y-x, y-x) d s \\
& \geq K\|y-x\|=2 \tilde{V}^{\prime}\left(\frac{\|y-x\|}{2}\right),
\end{aligned}
$$

where $\tilde{V}(z)=\frac{K}{2} z^{2}$ is the harmonic oscillator potential. Thus the fundamental gap on $\Omega$ is bounded below by that of the harmonic oscillator on the interval $[-D / 2, D / 2]$. This provides a sharp version of [Y3, Theorem 1.2]. The result also extends to entire domains: Suppose $V$ is a potential on $\mathbb{R}^{n}$ with $D^{2} V \geq K \delta_{i j}$. Then the eigenvalues $\lambda_{i}$ on $B_{R}(0)$ converge to those on $\mathbb{R}^{n}$, so the fundamental gap for this potential on $\mathbb{R}^{n}$ is no less than that of the one-dimensional harmonic oscillator, which is $\sqrt{2 K}$. Equality will hold in the limit of potentials of the form $V=\frac{K}{2}\left(x_{1}^{2}+n \sum_{i=2}^{n} x_{i}^{2}\right)$ as $n \rightarrow \infty$.

Our result also gives useful consequences for nonconvex potentials: Consider a one-dimensional potential $\tilde{V}$ which is even and has nonnegative third derivatives. A useful example is the double-well potential $\tilde{V}(z)=-a z^{2}+b z^{4}$ with $a, b>0$. Now consider higher-dimensional potentials of the form

$$
V(x)=\tilde{V}(\|x\|)+c \sum_{i=2}^{n} x_{i}^{2} .
$$

For large $c$ these are double-well potentials which agree with $\tilde{V}$ along the $x_{1}$-axis. One can check directly that $\tilde{V}^{\prime}$ is a modulus of convexity for $V$ for any $c \geq 0$, and it follows that the fundamental gap for such a potential on a convex domain $\Omega$ of diameter $D$ is bounded below by that of $\tilde{V}$ on the interval $[-D / 2, D / 2]$. Again, the result is sharp, with equality holding in the limit as $c \rightarrow \infty$ in the case where $\Omega$ contains the interval $(-D / 2, D / 2)$. 
An extension of our methods yields sharp results for domains in constant curvature spaces, implying that the spectral gap for a convex domain with convex potential is at least as large as the gap for a one-dimensional comparison problem (Equation (1) in $[\mathrm{Kr}]$ with Dirichlet boundary conditions), with equality in the limit of domains which are small neighbourhoods of a geodesic segment. We will provide the details elsewhere.

\section{REFERENCES}

[AC1] Ben Andrews and Julie Clutterbuck, Lipschitz bounds for solutions of quasilinear parabolic equations in one space variable, J. Differential Equations 246 (2009), no. 11, 42684283. MR2517770 (2010j:35237)

[AC2] - Time-interior gradient estimates for quasilinear parabolic equations, Indiana Univ. Math. J. 58 (2009), no. 1, 351-380. MR2504416 (2010k:35234)

[A] Mark S. Ashbaugh, The Fundamental Gap (2006), available at http://www.aimath.org/ WWN/loweigenvalues/.

[AB] Mark S. Ashbaugh and Rafael Benguria, Optimal lower bound for the gap between the first two eigenvalues of one-dimensional Schrödinger operators with symmetric single-well potentials, Proc. Amer. Math. Soc. 105 (1989), no. 2, 419-424. MR942630 (89f:81028)

[BK] Rodrigo Bañuelos and Pawel Kröger, Gradient estimates for the ground state Schrödinger eigenfunction and applications, Comm. Math. Phys. 224 (2001), no. 2, 545-550. MR1869875 (2002i:35135)

[BMH] Rodrigo Bañuelos and Pedro J. Méndez-Hernández, Sharp inequalities for heat kernels of Schrödinger operators and applications to spectral gaps, J. Funct. Anal. 176 (2000), no. 2, 368-399. MR.1784420 (2001f:35096)

[vdB] M. van den Berg, On condensation in the free-boson gas and the spectrum of the Laplacian, J. Statist. Phys. 31 (1983), no. 3, 623-637. MR.711491 (85i:82022)

[BL] Herm Jan Brascamp and Elliott H. Lieb, On extensions of the Brunn-Minkowski and Prékopa-Leindler theorems, including inequalities for log concave functions, and with an application to the diffusion equation, J. Functional Analysis 22 (1976), no. 4, 366-389. MR0450480(56:8774)

[D] Burgess Davis, On the spectral gap for fixed membranes, Ark. Mat. 39 (2001), no. 1, 65-74. MR1821082 (2002a:35165)

[GT] David Gilbarg and Neil S. Trudinger, Elliptic partial differential equations of second order, Classics in Mathematics, Springer-Verlag, Berlin, 2001. Reprint of the 1998 edition. MR.1814364 (2001k:35004)

[Ha] Evans M. Harrell, Double wells, Comm. Math. Phys. 75 (1980), no. 3, 239-261. MR.581948 (81j:81010)

[He] Antoine Henrot, Extremum problems for eigenvalues of elliptic operators, Frontiers in Mathematics, Birkhäuser Verlag, Basel, 2006. MR2251558 (2007h:35242)

[HP] Antoine Henrot and Michel Pierre, Variation et optimisation de formes: Une analyse géométrique, Mathématiques \& Applications (Berlin), vol. 48, Springer, Berlin, 2005. MR2512810(2009m:49003)

[Ho] Miklós Horváth, On the first two eigenvalues of Sturm-Liouville operators, Proc. Amer. Math. Soc. 131 (2003), no. 4, 1215-1224 (electronic). MR1948113 (2005e:34253)

[Ko] Nicholas J. Korevaar, Convex solutions to nonlinear elliptic and parabolic boundary value problems, Indiana Univ. Math. J. 32 (1983), no. 4, 603-614. MR703287 (85c:35026)

[Kr] Pawel Kröger, On the spectral gap for compact manifolds, J. Differential Geom. 36 (1992), no. 2, 315-330. MR.1180385 (94g:58236)

[La] Richard Lavine, The eigenvalue gap for one-dimensional convex potentials, Proc. Amer. Math. Soc. 121 (1994), no. 3, 815-821. MR1185270 (94i:35144)

[Li] Peter Li, A lower bound for the first eigenvalue of the Laplacian on a compact manifold, Indiana Univ. Math. J. 28 (1979), no. 6, 1013-1019. MR.551166 (81j:58084)

[LY] Peter Li and Shing Tung Yau, Estimates of eigenvalues of a compact Riemannian manifold, Geometry of the Laplace operator, Proc. Sympos. Pure Math., XXXVI, Amer. Math. Soc., Providence, R.I., 1980, pp. 205-239. MR.573435 (81i:58050) 
[L] Jun Ling, Estimates on the lower bound of the first gap, Comm. Anal. Geom. 16 (2008), no. 3, 539-563. MR2429968 (2009h:35087)

[PW] L. E. Payne and H. F. Weinberger, An optimal Poincaré inequality for convex domains, Arch. Rational Mech. Anal. 5 (1960), 286-292 (1960). MR.0117419 (22:8198)

[SWYY] I. M. Singer, Bun Wong, Shing-Tung Yau, and Stephen S.-T. Yau, An estimate of the gap of the first two eigenvalues in the Schrödinger operator, Ann. Scuola Norm. Sup. Pisa Cl. Sci. (4) 12 (1985), no. 2, 319-333. MR829055 (87j:35280)

[S] Robert G. Smits, Spectral gaps and rates to equilibrium for diffusions in convex domains, Michigan Math. J. 43 (1996), no. 1, 141-157. MR1381604 (97d:35037)

[Y1] Shing-Tung Yau, Gap of the first two eigenvalues of the Schrödinger operator with nonconvex potential, Mat. Contemp. 35 (2008), 267-285. MR2584188 (2010m:35097)

[Y2] _ Nonlinear analysis in geometry, Monographies de L'Enseignement Mathématique, vol. 33, L'Enseignement Mathématique, Geneva, 1986. Série des Conférences de l'Union Mathématique Internationale, 8. MR.865650 (88e:53001)

[Y3] _ An estimate of the gap of the first two eigenvalues in the Schrödinger operator, Lectures on partial differential equations, New Stud. Adv. Math., vol. 2, Int. Press, Somerville, MA, 2003, pp. 223-235. MR2055851 (2005c:35219)

[YZ] Qi Huang Yu and Jia Qing Zhong, Lower bounds of the gap between the first and second eigenvalues of the Schrödinger operator, Trans. Amer. Math. Soc. 294 (1986), no. 1, 341-349. MR819952 (87f:35179)

[ZY] Jia Qing Zhong and Hong Cang Yang, On the estimate of the first eigenvalue of a compact Riemannian manifold, Sci. Sinica Ser. A 27 (1984), no. 12, 1265-1273. MR794292 (87a:58162)

Mathematical Sciences Institute, Australian National University, Canberra, ACt 0200, Australia and Mathematical Sciences Center, Tsinghua University, Beijing, 100084, Peoples Republic of China and Morningside Center of Mathematics, Chinese Academy of Sciences, Beijing, 100190, Peoples Republic of China

E-mail address: Ben.Andrews@anu.edu.au

Centre for Mathematics and Its Applications, Australian National University, ACT 0200, Australia

E-mail address: Julie.Clutterbuck@anu.edu.au 